\title{
INNOVACIONES INTERNACIONALES EN ROBÓTICA MÉDICA PARA MEJORAR EL MANEJO DEL PACIENTE EN PERÚ
}

\author{
INTERNATIONAL INNOVATIONS IN MEDICAL ROBOTICS TO IMPROVE THE \\ PATIENT MANAGEMENT IN PERU
}

José Cornejo', Jorge A. Cornejo-Aguilar², J. Paul Perales-Villarroel, M.D. ${ }^{3}$

\begin{abstract}
RESUMEN
Surgical Engineering Society realizó una investigación, la primera revisión como pioneros en LATAM, desde el año 2015, que desarrolla el tema de las últimas tecnologías de robótica médica creadas en América del Norte, Europa y Asia, que se importan para aplicarlas en el sistema de salud de Perú y América Latina Los sistemas robóticos para cirugía, rehabilitación y asistencia se presentan cubriendo la descripción del diseño del control y las especificaciones técnicas. Además, el manejo del paciente mediante robots se analiza con herramientas de diagnóstico, pronóstico y tratamiento. El proceso para evaluar los nuevos inventos de los dispositivos robóticos y llevarlo al mercado se introduce donde en las tres etapas que se muestran a continuación: innovación, asuntos regulatorios y aplicación biomédica. Además, hay un alto porcentaje de población con discapacidad en América Latina, por lo tanto, es necesario que más científicos biomédicos se especialicen en robótica para comenzar a desarrollar nuevas ideas y mejorar el manejo del paciente. Finalmente, las nuevas tecnologías creadas en América Latina deben ser asequibles, simples y eficientes para apoyar la situación económica de la atención médica en el continente y brindar los mejores estándares de calidad para mejorar los resultados del tratamiento en los pacientes.
\end{abstract}

Palabras clave: Surgical Engineering Society; Robótica Médica; Sistema de Salud; Perú; América Latina; Cirugía; Rehabilitación; Asistencia; Manejo de Pacientes; Diagnóstico; Pronóstico; Tratamiento. (fuente: DeCS BIREME)

\begin{abstract}
Surgical Engineering Society conducted a research, the First Review as the pioneers in LATAM, since year 2015, which develops the topic of the latest Medical Robotics technologies created in North América, Europe and Asia, which are imported to apply in the Healthcare System of Peru and Latin America. The robotic systems for surgery, rehabilitation and assistance are presented covering the description of control design and technical specifications. Besides, the patient management using robots is analyzed with diagnosis, prognosis and treatment tools. The process to evaluate the new inventions of robotic devices and bring to the market is introduced where three stages are shown as the following: innovation, regulatory affairs and biomedical application. Moreover, there is a high percentage of population with disability in Latin America, therefore, it is necessary that more biomedical scientists be specialized in robotics to start developing new ideas to improve the patient management. Finally, the new technologies created in Latin America must be affordable, simply and efficient in order to support the healthcare economic situation in the continent and give the best quality standards to improve treatment outcomes on patients.
\end{abstract}

Key words: Surgical Engineering Society; Medical Robotics; Healthcare System; Peru; Latin America; Surgery; Rehabilitation; Assistance; Patient Management; Diagnosis; Prognosis; Treatment. (source: MeSH NLM)

\footnotetext{
${ }^{1}$ MSc. Biomedical Eng. Program - VIU, Valencian International University Surgical Engineering Society - CEO, Lima-Perú

${ }^{2}$ Faculty of Human Medicine URP, Surgical Engineering Society - VP, Lima-Perú.

${ }^{3}$ General Surgery Resident Kendall Regional Medical Center Miami, FL, United States.
}

Citar como: José Cornejo, Jorge A. Cornejo-Aguilar, J. Paul Perales-Villarroel, M.D. Innovaciones internacionales en robótica médica para mejorar el manejo del paciente en Perú. Rev. Fac. Med. Hum. Octubre 2019; 19(4):105-113. DOI 10.25176/RFMH.v19i4.2349 


\section{INTRODUCCIÓN}

El rápido progreso de la técnica robótica y la curva de aprendizaje en los procedimientos médicos ofrece nuevas oportunidades para la ingeniería biomédica y sanitaria'.

La invención, la innovación en el desarrollo tecnológico no es una ciencia simple. La innovación en la asistencia sanitaria debe seguir evolucionando a medida que la tecnología y los recursos sean accesibles; sin embargo, la innovación realmente requiere cierto nivel de riesgo en el manejo del paciente porque es importante que el dispositivo robótico pueda cumplir con las expectativas del paciente. Es posible que los médicos y los centros médicos no se sientan cómodos con el mayor riesgo asociado con los dispositivos médicos innovadores; existen procedimientos bien establecidos para validar una nueva herramienta / dispositivo médico ${ }^{2}$. Por lo tanto, los investigadores establecen algunos parámetros para realizar un proceso exitoso de investigación, desarrollo e innovación en el campo de la robótica médica, como se muestra en la Tabla 1.

Tabla 1. Investigación, desarrollo e innovación.

\begin{tabular}{ccccc} 
& & Robótica Médica & \\
Aspectos de control & Posicionamiento & Orientación de Trayectorias & Control de la fuerza \\
Ajuste & En el espacio & A tiempo & Dar forma & $\begin{array}{c}\text { Al estado del } \\
\text { paciente }\end{array}$ \\
\hline
\end{tabular}

Al presentar la robótica médica en América Latina, es importante afirmar que esas tecnologías tienen 10 años de desarrollo e investigación, pero se necesitan investigadores más especializados para iniciar centros de innovación con el fin de crear nuevas tecnologías para aplicaciones quirúrgicas y de rehabilitación.
En América del Norte, Europa y Asia, la investigación en dispositivos y robótica médica está muy avanzada. En América Latina, el procedimiento de flujo para introducir un robot para aplicaciones quirúrgicas y de rehabilitación es el siguiente que se muestra en la Fig. $1 .^{2}$, donde se sugiere la información presentada para hacer más patentes en la industria de la salud.

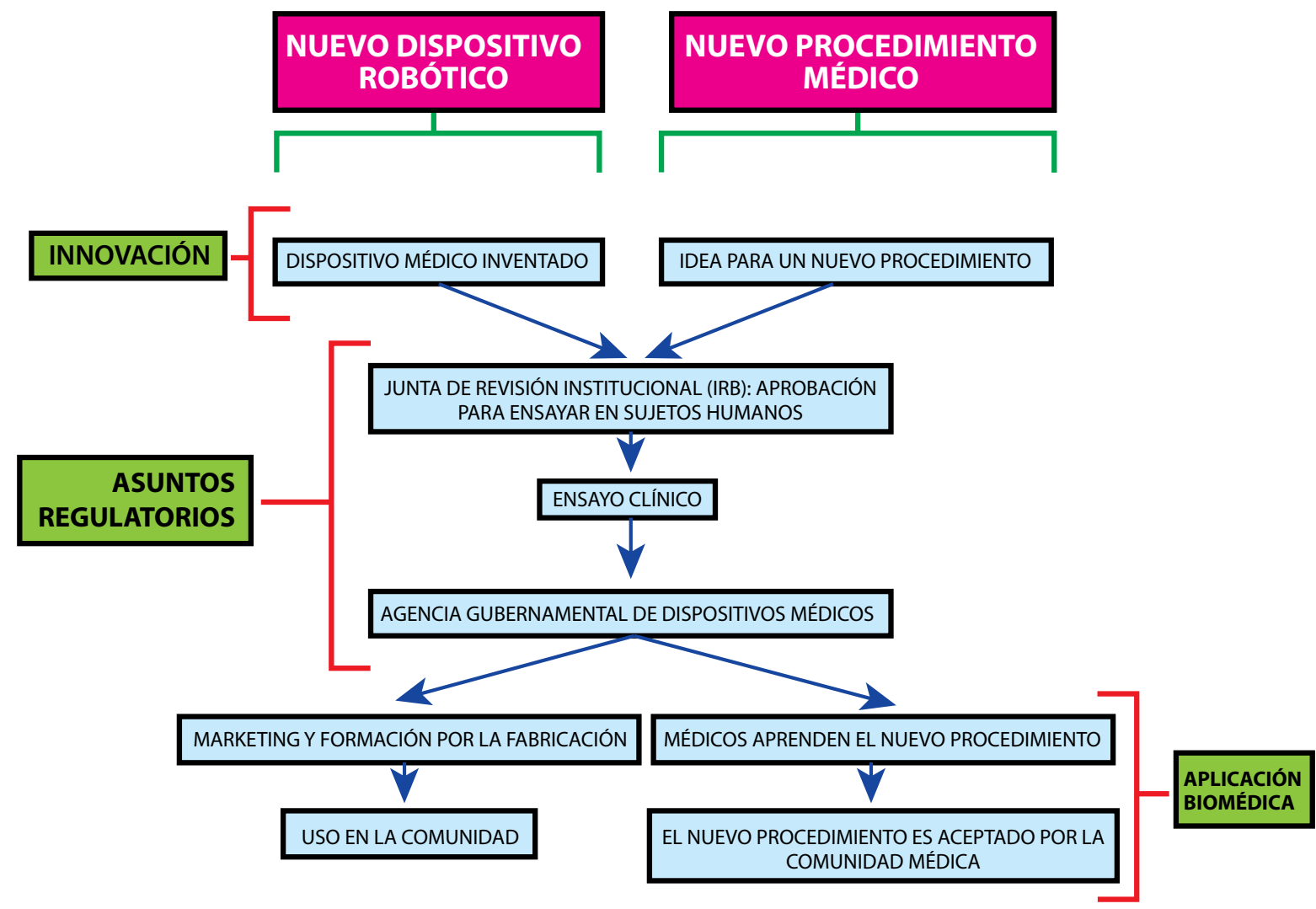

Figura 1. Diagrama de dispositivo médico robótico e innovación de procedimientos. 


\section{MANEJODELPACIENTECONROBÓTICA} MÉDICA

La innovación internacional en un campo competitivo y en rápida evolución, como la cirugía, la rehabilitación y la asistencia, requieren que la comunidad esté dispuesta a cambiar la comodidad del procedimiento por la oportunidad de mejorar los resultados. Por lo tanto, los profesionales de la salud han estado apoyando en el diagnóstico, el pronóstico y el tratamiento con la aplicación de la robótica médica en algunas patologías con el fin de mejorar la esperanza de vida en la población mundial, por lo que en la Tabla II se adapta las herramientas más comunes para hacer una adecuada manipulación del paciente ${ }^{3}$.

Tabla 2. El manejo del paciente.

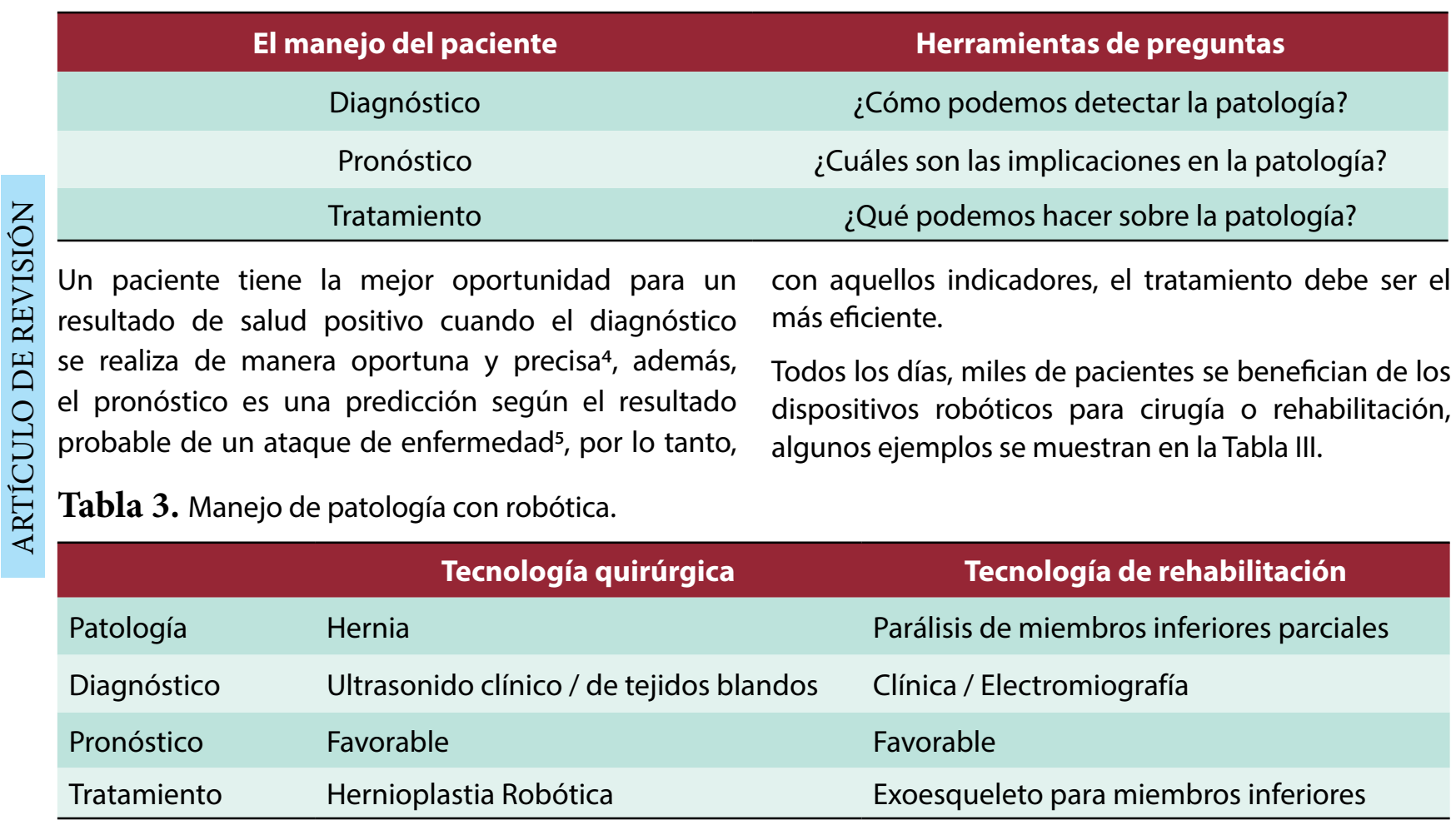

En América Latina, la robótica médica se importa de compañías internacionales para aplicarla en el cuidado de la salud, porque no hay demasiadas tecnologías avanzadas patentadas para crear un proyecto de innovación inicial para investigar y desarrollar, y también los países carecen de grandes cantidades de dinero para financiamiento. Los primeros sistemas robóticos quirúrgicos importados en América Latina fueron en México y Brasil, por lo que años más tarde, en 2016, los cirujanos realizaron una investigación para analizar qué especialidades en cirugía robótica son las más utilizadas, como cirugía general, urología y ginecología como se muestra en la Fig. $2^{6}$.

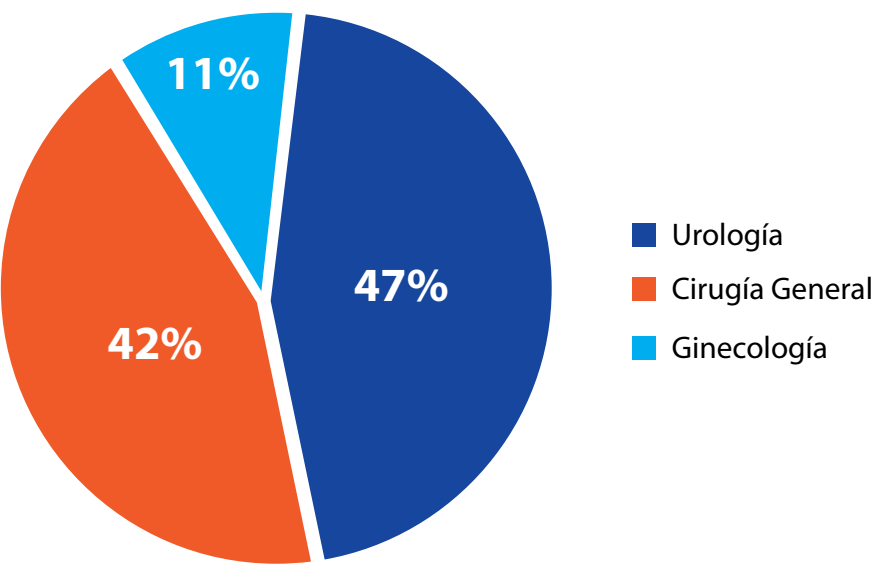

Figura 2. Porcentaje de procedimientos quirúrgicos robóticos. 
Además, hay un informe sobre el porcentaje de (31.28\%) con discapacidad es Perú, lo que demuestra discapacidad en América Latina presentado en 2006, que América Latina tiene una baja tasa de rehabilitación donde el país con la mayor cantidad de población de alta calidad, esa información se muestra en la Fig. ${ }^{7}$.

\section{Discapacidad en América Latina}

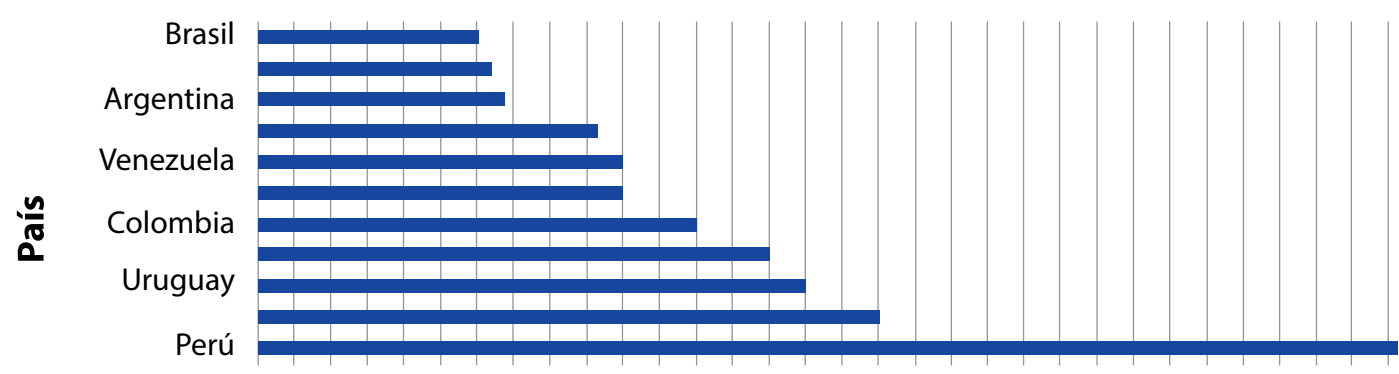

$0 \begin{array}{llllllllll} & 2 & 3 & 4 & 5 & 6 & 7 & 8 & 9 & 1011121314151617181920212223242526272829303132\end{array}$

\begin{tabular}{|l|c|c|c|c|c|c|c|c|c|c|c|}
\cline { 2 - 10 } \multicolumn{1}{l|}{} & Perú & Guatemala & Uruguay & Honduras & Colombia & Mexico & Venezuela & Costa Rica & Argentina & Chile & Brasil \\
\hline $\begin{array}{l}\text { Porcentaje de la } \\
\text { población total }\end{array}$ & 31.28 & 17 & 15 & 14 & 12 & 10 & 10 & 9.32 & 6.8 & 6.39 & 6 \\
\hline
\end{tabular}

Figura 3. Valores de porcentaje de discapacidad en América Latina.

\section{TECNOLOGÍAS BIOMÉDICAS Y ROBÓTICAS}

\section{A. Robótica Quirúrgica}

Esta tecnología se enfoca en el área de aplicaciones mínimamente invasivas que se estableció a fines de la década de 1990, también tiene características ergonómicas y de teleoperación. Esos procedimientos quirúrgicos se han llevado a cabo en más de 3 millones de pacientes hasta la fecha ${ }^{8}$. La robótica quirúrgica ha recibido una gran atención por parte de los sistemas de salud en el mundo y la industria. Representa un cambio radical en el trabajo de la sala de operaciones, convirtiendo al robot en un miembro del equipo quirúrgico.

Cuando se considera al robot como otro miembro, el robot se percibe como un "cirujano robótico inteligente", con autonomía y capacidad de toma de decisiones. Sin embargo, es más preciso y más cercano a la realidad ver al robot quirúrgico como una "herramienta quirúrgica avanzada", es decir, como un dispositivo colocado en las manos del cirujano que le permite aumentar sus capacidades, hacerlo más preciso, mitigar los problemas de fatiga, mejorar la destreza, etc. De esta manera, la autonomía de los robots quirúrgicos está muy limitada a ciertas tareas específicas, mientras que la mayoría de las veces el robot es una herramienta que funciona bajo el esquema de control teleoperado?.

El diseño del robot quirúrgico debe incluir un análisis de riesgo ${ }^{10}$, porque el dispositivo tiene contacto con el humano durante la etapa de anestesia y podría ser peligroso. Además, en todo el mundo, para crear la ingeniería de control del sistema es común la aplicación del Diagrama de bloques como se muestra en la Fig. $4 .{ }^{11}$, que brinda seguridad, precisión, comodidad y facilidad de uso para el cirujano.

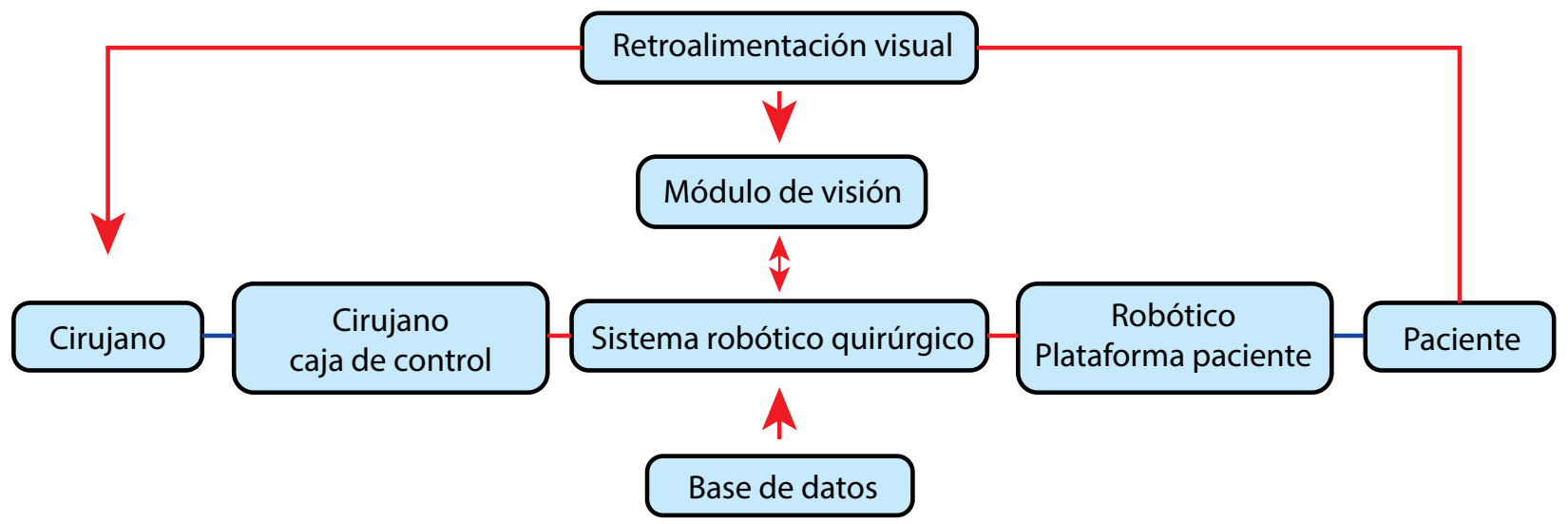

Figura 4. Diagrama de bloques del sistema robótico quirúrgico - Control. 
La cirugía asistida por computadora (CAS) es un proceso global que abarca varias etapas; diagnóstico, planificación, intervención, etapas postoperatorias. Es importante mejorar cada uno de ellos para mejorar el proceso general ${ }^{12}$. La navegación quirúrgica permite la localización en 3D de los diferentes indicadores involucrados en el procedimiento quirúrgico; como herramientas quirúrgicas, campo quirúrgico e información anatómica del paciente (imágenes preoperatorias o intraoperatorias) ${ }^{13}$.

Se podrían hacer diferentes clasificaciones de robots para cirugía, dependiendo de su esquema de control (autónomo o teleoperado), o su posición con respecto al paciente (robot montado en mesa o robot montado en paciente), o su arquitectura mecánica (robot serie, robot paralelo o móvil) ${ }^{14}$.
En todo el mundo, el sistema de cirugía robótica más utilizado es el da Vinci ${ }^{\circledR}$, tiene ventajas en comparación con la cirugía laparoscópica, como menos pérdida y necesidad de transfusión de sangre, menos dolor postoperatorio, hospitalizaciones y tiempos de recuperación más cortos; a expensas de tiempos de operación más largos y un costo más alto en comparación con la técnica laparoscópica ${ }^{15}$.

En América Latina, 2018, el país que tiene la mayoría de daVinci System ${ }^{\circledR}$ es Brasil con 37 robots, y es importante presentar que Perú no tiene ningún sistema quirúrgico para aplicar en el sistema de salud ${ }^{15}$, así que a través de este artículo de investigación queremos alentar a importar la máquina quirúrgica con el propósito de mejorar el manejo del paciente para la cirugía, como se muestra en la figura 5 .

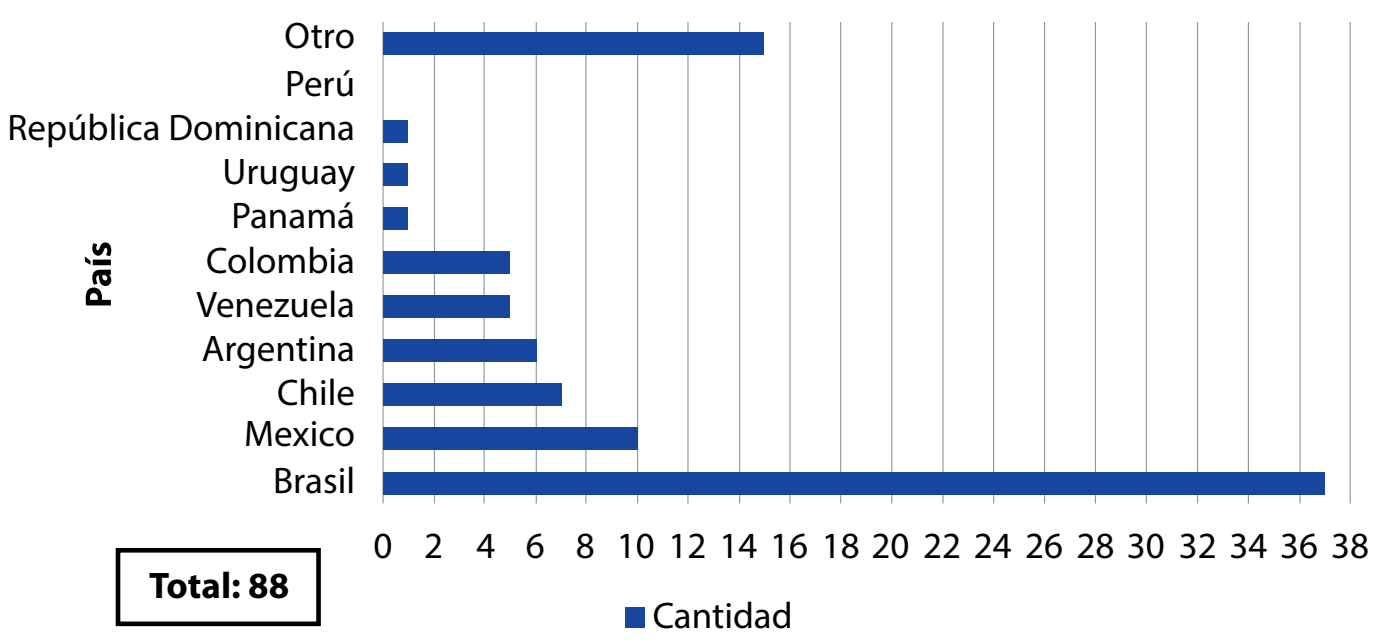

Figura 5. Cantidad de sistema robótico quirúrgico da Vinci ${ }^{\circledR}$ - América Latina.

En todo el mundo, hay algunas compañías que en la Tabla IV. En América Latina aún no se ha inventado desarrollan robótica quirúrgica para aplicar en partes un robot quirúrgico que se use directamente en anatómicas específicas del cuerpo, como se muestra procedimientos con humanos.

Tabla 4. Tecnología de robótica quirúrgica - sección anatómica.

\section{Anatomía}

\begin{tabular}{lllll} 
Abdomen & Intuitive Surgical & TransEnterix & CMR Surgical & SS Innovations \\
\hline Neuro & Medtronic & Synaptive & Zimmer & Monteris \\
\hline Keep/Hip & Stryker/Mako & THINK Surgical & Smith \& Nephew & Zimmer Biomet \\
ENT & Medrobotics & Intuitive Surgical & Medineering & Galen Robotics \\
Spine & Medtronic/Mazor & Globus Medical & Zimmer Biomet & NuVasive \\
Ojos & Consultores de Cambridge & Preceyes & & \\
Corazón & Stereotaxis & Heartlander Surgical & \\
Livianos & J\&/Auris & Intuitive Surgical & \\
Piel & Avra Medical Robotics & & \\
Uretra & Procept BioRobotics & & \\
\hline
\end{tabular}


En Perú, hay un Proyecto de Innovación llamado "Biomedik Surgeon ${ }^{\circledR}$, desarrollado por el Ing. José Cornejo y el Dr. Jorge A. Cornejo-Aguilar, como el primer prototipo de sistema robótico quirúrgico inventado en el país para entrenamiento y simulación utilizado por estudiantes de medicina ${ }^{16}$, como se muestra en la Fig. 6.

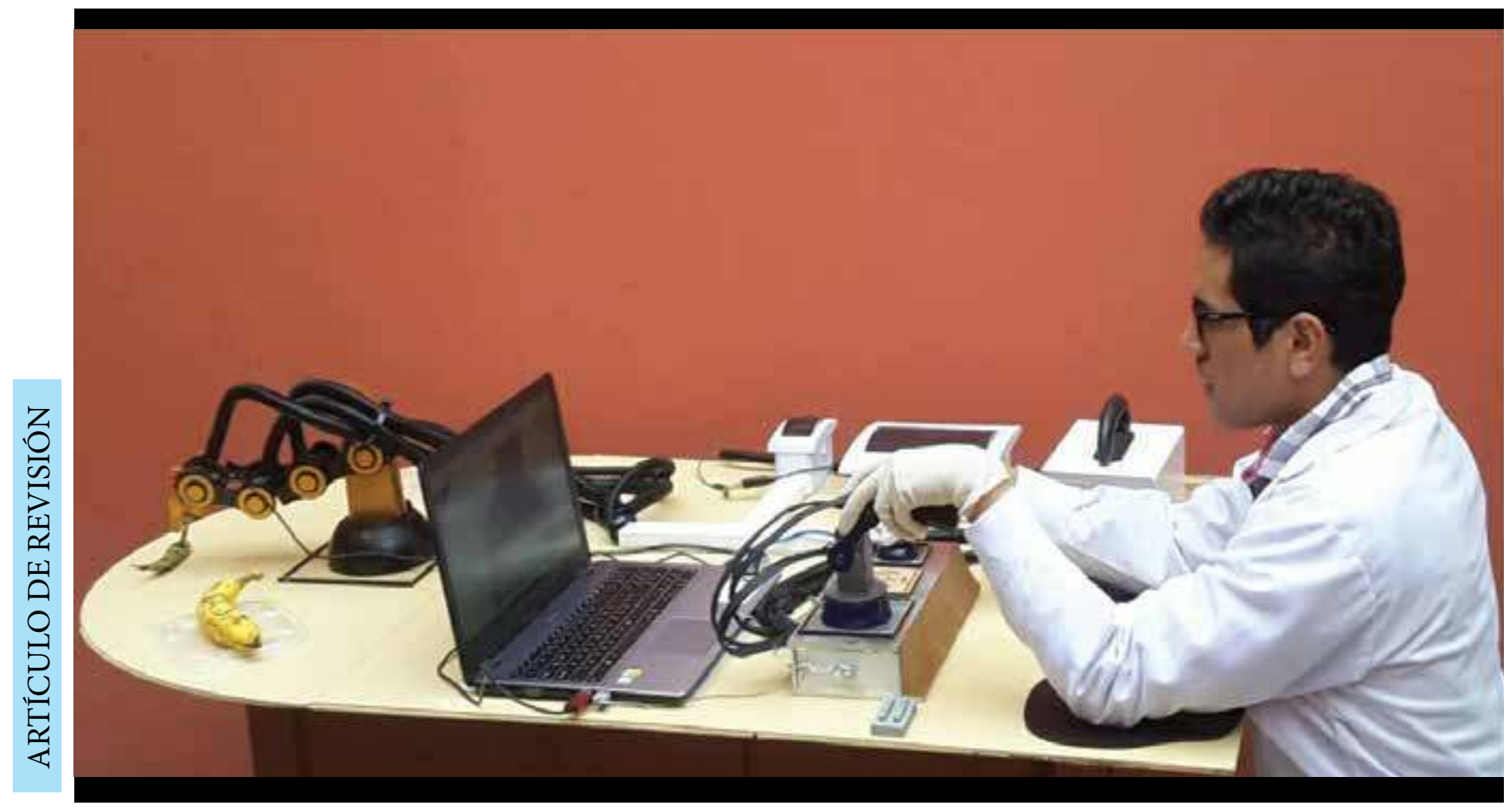

Figura 6. Eng. José Cornejo and Biomedik Surgeon ${ }^{\oplus}$.

\section{B. Robótica para Rehabilitación y Asistencia}

Esta tecnología es un campo interdisciplinario emergente donde la robótica, los sensores y la retroalimentación se utilizan de formas novedosas para reaprender, mejorar o restaurar los movimientos funcionales en humanos. Involucra el caminar, donde el sistema neuromuscular orquesta la marcha, la postura y el equilibrio, también afecciones como accidente cerebrovascular, déficit vestibular o vejez que deterioran esta importante actividad. Además, el entrenamiento robótico, la rehabilitación de la marcha y las ortesis cooperativas describen los trabajos actuales en el campo para abordar este problema, llevar la rehabilitación desde la clínica al entorno doméstico, de modo que estas ayudas médicas estén más fácilmente disponibles para los usuarios ${ }^{17}$.

La robótica de rehabilitación ofrece oportunidades considerables para mejorar la calidad de vida de las personas con discapacidad física, provocando movimientos pasivos repetitivos de la extremidad del paciente sostenido por el robot, sustituyendo o apoyando los movimientos realizados por el fisioterapeuta ${ }^{18}$. Entonces, la interfaz hombremáquina alcanza su máximo exponente, una de estas tecnologías se llama Realidad Virtual, que permite al usuario interactuar y sumergirse en un entorno generado por computadora de forma natural ${ }^{19}$. Sin embargo, las terapias estándar proporcionan beneficios en términos de movilidad mejorada ${ }^{20}$.

En todo el mundo, los científicos han creado, utilizando ingeniería de control, un diseño estandarizado común del sistema utilizando el Diagrama de bloques, como se muestra en la Fig. $7 .{ }^{17}$, que es un sistema de circuito cerrado que combina la fuerza, la posición, la velocidad y la aceleración con sensores y actuadores acoplados a ortesis o prótesis, lo que ayuda a evaluar la evolución de la terapia en el paciente. 


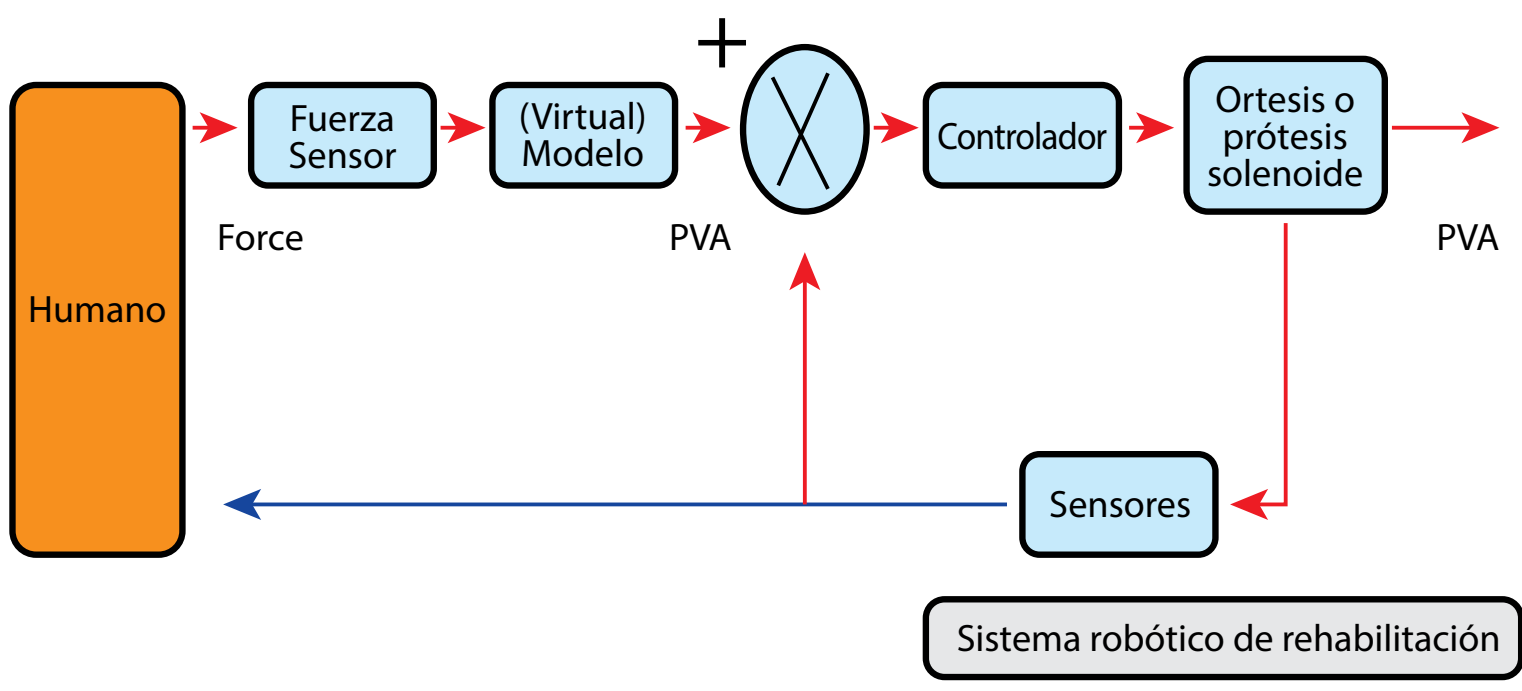

* PVA: posición, velocidad, aceleración

Figura 7. Diagrama de bloques del sistema robótico de rehabilitación - Control.

Se podrían hacer diferentes clasificaciones de robots para rehabilitación, dependiendo de los robots basados en efectores finales y el exoesqueleto, estos son: robots para rehabilitación de miembros superiores, miembros inferiores y extremidades distales.

Existe otro concepto llamado Robótica Asistencial, que es un dispositivo tecnológicoadaptativo que interactúa directamente con el usuario para mantener y / o aumentar las capacidades funcionales de las personas, a través de sensores, actuadores y procesamiento ${ }^{21}$, con el fin de apoyar al paciente en las áreas asociadas. con actividades psicomotoras de rutina con robots autónomos y robots cuyas funcionalidades son compartidas con el usuario, utilizadas con fines domésticos, de movilidad, hospitalidad y terapéuticos. Diseñar una prótesis robótica y una ortesis requiere la combinación de mecatrónica, neurociencia, ingeniería eléctrica, ciencia cognitiva, procesamiento de señales, diseño de baterías, nanotecnología y ciencias de la conducta ${ }^{17}$, con el objetivo de mejorar el proceso de recuperación y facilitar la restauración del físico. funcionar mediante la entrega de entrenamiento de alta dosis y alta intensidad ${ }^{22}$.

A nivel internacional, hay algunos centros de tecnología médica que han creado robots de rehabilitación para mejorar la calidad de vida de los pacientes que han sufrido daños en su sistema nervioso-motorneurológico $\frac{23}{}$, esa descripción se muestra en la Tabla 5.

Tabla 5. Tecnología de rehabilitación y robótica asistiva.

\begin{tabular}{ll} 
MySpoon & Permita comer con la mínima ayuda del cuidador \\
\hline MIT Manus & Para terapia física de víctimas de derrame cerebral \\
\hline ARMin & Dispositivo exoesquelético unido al brazo \\
\hline Anklebot & Dispositivo exoesquelético unido a la pierna \\
\hline GENTLE/s & Asistencia robótica en rehabilitación neurológica y motora
\end{tabular}

En Perú, existe la empresa "Biom3D", administrada por el Ing. Cesar Martel y el Ing. Carol Sandoval, que desarrolla prótesis para niños de hogares y comunidades con bajo recursos como una iniciativa social llamada "Manos en Acción", como se muestra en la Fig. 8. 


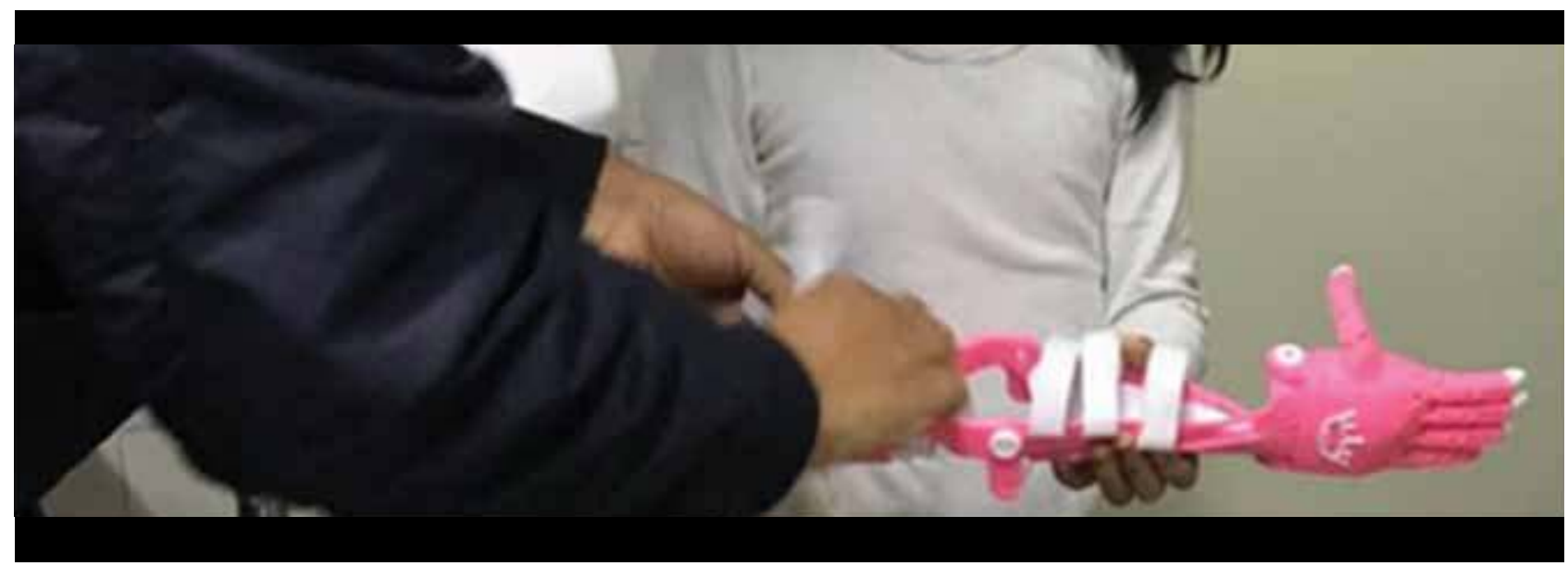

Figura 8. Biom $3 \mathrm{D}^{\circledR}$, Manos en Acción - Arm Prosthesis.

\section{CONCLUSIÓN}

El uso de la robótica médica para la salud es muy importante para poder apoyar la atención médica del paciente y controlar la patología utilizando un diagnóstico, un pronóstico y un tratamiento de alto nivel sobresalientes con la finalidad de obtener una recuperación exitosa.

La cirugía, la rehabilitación y la asistencia son las principales áreas de aplicación de la robótica en la asistencia sanitaria. Recientemente, aproximadamente en los últimos 15 años, América Latina tiene acceso para importar las últimas tecnologías desarrolladas en América del Norte, Europa y Asia. Sin embargo, es importante que los países de esta parte del continente comiencen a invertir en las nuevas tecnologías médicas asequibles y eficientes para establecer nuevas formas de dar una mejor calidad de vida a la población.

Los deberes del ingeniero biomédico con especialización en robótica médica son tan importantes e indispensables porque impulsan las herramientas más efectivas para analizar los requisitos médicos propuestos por los profesionales de la salud de acuerdo con las necesidades del paciente, y también pueden trabajar como un equipo quirúrgico mejorando los resultados de salud y mejorando la seguridad del paciente.

Contribuciones de autoría: Los autores participaron en la génesis de la idea, diseño de proyecto, recolección, análisis de la información y preparación del manuscrito del presente trabajo de investigación.

Financiamiento: Autofinanciado.

Conflicto de interés: Los autores declaran no tener conflicto de interés en la publicación de este artículo.

Recibido: 19 de junio del 2019

Aprobado: 03 de setiembre del 2019

Correspondencia: Jorge A. Cornejo Aguilar

Dirección: Calle Galicia 369. Lima, Perú.

Teléfono: +51993519893

Correo:jcornejo_17@ieee.org 


\section{REFERENCIAS BIBLIOGRÁFICAS}

1. Hu, C., Shi, Q., Liu, L., Wejinya, U., Hasegawa, Y., \& Shen, Y. "Robotics in Biomedical and Healthcare Engineering". Journal of healthcare engineering, 2017, 1610372.doi:10.1155/2017/1610372

2. Vyas, D., \& Cronin, S. "Peer Review and Surgical Innovation: Robotic Surgery and Its Hurdles". American journal of robotic surgery, 2(1), 39 44, 2015. doi:10.1166/ajrs.2015.1018

3. Wray, C. M., \& Loo, L. K., "The Diagnosis, Prognosis, and Treatment of Medical Uncertainty". Journal of graduate medical education, 7(4),523527, 2015. doi:10.4300/JGME-D-14-00638.1

4. Holmboe, Eric S., and Steven J. Durning. "Assessing Clinical Reasoning: Moving from in Vitro to in Vivo." Diagnosis (Berlin, Germany) 1 (1): 111 $17,2014$.

5. Committee on Diagnostic Error in Health Care; Board on Health Care Services; Institute of Medicine; The National Academies of Sciences, Engineering, and Medicine; Balogh EP, Miller BT, Ball JR, editors "Improving Diagnosis in Health Care". Washington (DC): National Academies Press (US); 2015 Dec 29. 2, The Diagnostic Process. Available from: https://www.ncbi.nlm.nih.gov/books/NBK338593/

6. Ruiz Suárez, Eduardo Jafet, Rodrigo Ortiz Wong, Gamaliel Rodríguez Reyes, David Valadez Caballero, Jorge Alberto Blanco Figueroa, and Juan Roberto González Santamaría. "Experiencia Inicial En Cirugía Robótica Mínimamente Invasiva En Hospital de Tercer Nivel En México." Revista Mexicana de Cirugía Endoscópica 17 (1): 29-33, 2016

7. Alicia Amate, E. "Discapacidad: lo que todos debemos saber", Pan American Health Org, 2006. Available from: https://play.google.com/ store/books/details?id=bnf6zhhwfDQC

8. Desai, Jaydev P., Rajni V. Patel, Antoine Ferreira, and Sunil Agrawal. "The Encyclopedia of Medical Robotics." The Encyclopedia of Medica Robotics. 2018. https://doi.org/10.1142/10770-vol1.

9. Bekey, George, "Springer Handbook of Robotics (B. Siciliano and O. Khatib; 2008) [Book Review]." IEEE Robotics \& Automation Magazine. 2008. https://doi.org/10.1109/mra.2008.928399.

10. Kazanzides, P., Fichtinger, G., Hager, G., Okamura, A. M., Whitcomb, L. L., \& Taylor, R. H., "Surgical and interventional robotics - Core concepts, technology, and design". IEEE Robotics and Automation Magazine, 15(2), 122-130, 2008. https://doi.org/10.1109/MRA.2008.926390

11. Najarian, Siamak, Javad Dargahi, Goldis Darbemamieh, and Siamak Hajizadeh Farkoush. "Mechatronics in Medicine A Biomedica Engineering Approach". 1st ed. McGraw-Hill Professional, 2011. ISBN:0071768963 9780071768962
12. Vanja Bozovic, I-Tech, "Medical Robotics”, 2008. ISBN 978-3-902613-18-9.

13. Jens-Uwe Stolzenburg, I.A. Turk and E.N. Liatsikos, "Laparoscopic and Robot-Assisted Surgery in Urology", Springer, 2011. ISBN 978-3642-00890-0.

14. J. Rosen, B. Hannaford, R.M. Satava, "Surgical Robotics. Sytems Applications and Visions". Springer, 2011. ISBN 978-1-4419-1125-4.

15. Jorge A. Cornejo-Aguilar, José Cornejo, Mariela Vargas, Rau Sebastian. "La revolución de la cirugía robótica en latino américa y la futura implementación en el sistema de salud del Perú". [Cartas al Editor]. 2019; 19(1):108-111. (Enero 2018). DOI 10.25176/RFMH.v19. $\mathrm{n} 1.1800$

16. J. Cornejo, J. A. Cornejo-Aguilar and R. Palomares, "Biomedik Surgeon: Surgical Robotic System for Training and Simulation by Medical Students in Peru," 2019 International Conference on Control of Dynamical and Aerospace Systems (XPOTRON), Arequipa, Peru, 2019, pp. 1-4. doi: 10.1109/XPOTRON.2019.8705717

17. Opensurg, C."ROBOTICA MEDICA Notas Prácticas Para El Aprendizaje de La Robótica En Bioingenierı." CYTED, 2013.

18. G. Bolmsjo, H. Neveryd and H. Eftring, "Robotics in rehabilitation", IEEE Transactions on Rehabilitation Engineering, vol. 3, no. 1, pp. 77-83. 1995. doi: $10.1109 / 86.372896$

19. M. T Schultheis and A. A Rizzo, "The application of virtual reality technology in rehabilitation", Rehabilitation Psychology 46, no. 5, 296 $-311,2011$

20. G. Saposnik, "Virtual reality in stroke rehabilitation a meta-analysis and implications for clinicians", Journal of the American Heart Association 42, no. 5, 1380 - 1386, 2011

21. E Henneman and L. M. Mendell, "Functional organization of the motoneuron pool and its inputs", Handbook of physiology: The nervous system, American Physiological Society, 423-507, 1981.

22. Siciliano, Bruno, and Oussama Khatib. 2016. "Robotics and the Handbook." Springer Handbook of Robotics. https://doi. org/10.1007/978-3-319-32552-1_1.

23. Laut, J., Porfiri, M., \& Raghavan, P., "The Present and Future of Robotic Technology in Rehabilitation". Current physical medicine and rehabilitation reports, 4(4), 312-319, 2017. doi:10.1007/s40141-0160139-0

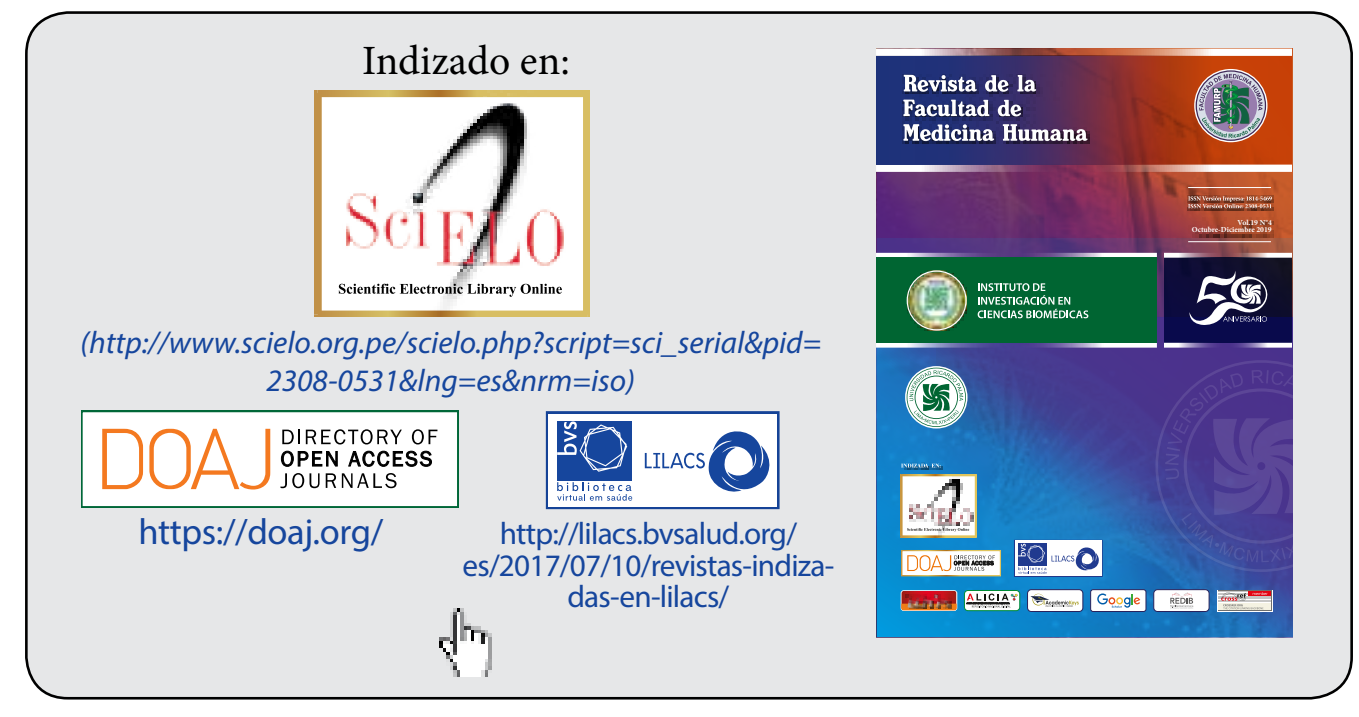

